\title{
Search for Astrophysical Tau Neutrinos in Six Years of High-Energy Starting Events in IceCube
}

\author{
The IceCube Collaboration ${ }^{\dagger}$ \\ $\dagger$ http://icecube.wisc.edu/collaboration/authors/icrc17_icecube \\ E-mail: marcel.usnereicecube.wisc.edu
}

\begin{abstract}
The IceCube Neutrino Observatory at the geographic South Pole is a cubic kilometer Cherenkov detector built to measure high-energy neutrinos from cosmic sources. It has reported the detection of a diffuse flux of astrophysical neutrinos in the energy range from $\sim 10 \mathrm{TeV}$ to $\sim 10 \mathrm{PeV}$ consistent with a neutrino flavor ratio of $v_{\mathrm{e}}: v_{\mu}: v_{\tau} \simeq 1: 1: 1$ as expected from pion decay in astrophysical sources after propagation to Earth. However, no tau neutrino has been identified so far. Its observation would be a smoking gun for astrophysical neutrinos and constrain their possible sources. The double bang channel is most promising for identifying tau neutrino interactions. Its event signature is unique to the tau flavor, linking two consecutive particle showers from the charged current interaction of a tau neutrino with an ice nucleus and the subsequent decay of the produced tau lepton. It can only be well resolved at deposited energies above a few $100 \mathrm{TeV}$ where the average tau decay length is larger than $20 \mathrm{~m}$. Results are presented from an analysis which uses an optimized direct reconstruction of the double bang event signature using six years of high-energy starting events (HESE) in IceCube. It is the most recent search for tau neutrinos allowing a measurement of the high-energy flavor ratio which, for the first time, is sensitive to the tau neutrino fraction.
\end{abstract}

Corresponding authors: $M$. Usner*1

${ }^{1}$ DESY, Platanenallee 6, D-15738 Zeuthen, Germany

35th International Cosmic Ray Conference - ICRC2017

10-20 July, 2017

Bexco, Busan, Korea

${ }^{*}$ Speaker. 


\section{Introduction}

The IceCube Neutrino Observatory at the South Pole is a Cherenkov detector built to measure high-energy neutrinos from cosmic sources. A volume of $\sim 1 \mathrm{~km}^{3}$ of the Antarctic ice is instrumented with 5160 digital optical modules (DOMs). Neutrinos are observed via Cherenkov light emitted by secondary particles produced in deep-inelastic neutrino-nucleon interactions. Details about the detector instrumentation can be found in [1]. The IceCube collaboration has reported a diffuse flux of astrophysical neutrinos in the energy range from $\sim 10 \mathrm{TeV}$ to $\sim 10 \mathrm{PeV}$ using multiple years of operation $[2,3,4,5]$. It is consistent with an observed flavor ratio of $\gamma_{\mathrm{e}}: \gamma_{\mu}: \nu_{\tau}=1: 1: 1$, however no tau neutrino interaction has been identified so far. IceCube has observed many tracklike events which can be readily distinguished from cascade-like events. While track-like events are generated in $v_{\mu}$ interactions, cascade-like events may be generated in both $v_{\mathrm{e}}$ and $v_{\tau}$ as well as neutral current interactions. Therefore, the knowledge about the tau neutrino fraction is largely unconstrained since no tau neutrino interaction could be identified so far.

An observation of a tau neutrino interaction in IceCube would be interesting for three reasons. First, it could significantly improve the measurement precision of the astrophysical flavor ratio and thus constrain possible production mechanisms and sources of cosmic neutrinos. The expected ratio of $\sim 1: 1: 1$ at Earth arises from the assumption that neutrinos are produced in the decays of charged pions and secondary muons leading to a flavor ratio of $1: 2: 0$ at the source [6]. There are other source scenarios like the muon-damped case with $0: 1: 0$ and the neutron-beam case with $1: 0: 0$ at the source leading to detectable flavor ratios at Earth that deviate from the usually modeled $1: 1: 1$ expectation $[7,8]$. Second, atmospheric tau neutrinos from cosmic ray air showers are strongly suppressed. In the relevant energy range above $100 \mathrm{TeV}$ the atmospheric neutrino flux is expected to be dominated by a charm component of which almost entirely only the $\mathrm{D}_{\mathrm{s}}$ decay produces detectable tau neutrinos. The flavor ratio of the prompt neutrino flux is expected to be of the order of $\sim 1: 1: 0.1$. Taking current upper limits on a prompt neutrino flux into consideration [9], the observation of a tau neutrino event around $1 \mathrm{PeV}$ in IceCube is $\sim 100$ times more likely to be of astrophysical than of atmospheric origin. Third, only few tau neutrinos have ever been detected in dedicated accelerator experiments and none at energies accessible to IceCube $[10,11]$.

There are many different signatures of tau neutrino interactions in IceCube. The most interesting signature is the "double bang" (or "double cascade"). A charged current tau neutrino interaction creates a hadronic cascade and a tau lepton that decays into a hadronic or an electromagnetic cascade, thereby linking two subsequent cascades. It accounts for $\sim 59 \%$ of all tau neutrino interactions. The mean decay length of the tau lepton scales with its energy and is on average $\sim 50 \mathrm{~m}$ per PeV tau energy. Therefore, most double cascade events at energies below $\sim 100 \mathrm{TeV}$ cannot be resolved in IceCube and effectively look like single cascades.

Without tau neutrino identification, previous all-flavor ratio measurements were only based on distinguishing track-like from cascade-like events and thus showed a large degeneracy between the electron- and tau-neutrino fractions $[12,13]$. The most recent direct search for tau neutrino interactions in IceCube has seen no events and set an integrated upper limit on the tau neutrino flux of $\phi_{v_{\tau}}\left(E_{\gamma_{\tau}}\right)<5.1 \cdot 10^{-18}\left(E_{\gamma_{\tau}} / 100 \mathrm{TeV}\right)^{-2} \mathrm{GeV}^{-1} \mathrm{~cm}^{-2} \mathrm{sr}^{-1} \mathrm{~s}^{-1}$ [14]. A combination of both results discussed in [13] and [14] gave the most stringent all-flavor ratio measurement including the most sensitive constraint on the tau neutrino fraction so far [15]. 
The analysis presented here introduces a new identification method of tau neutrino interactions in IceCube by explicitly reconstructing events with a double cascade hypothesis. It aims at the discovery of the first tau neutrino interaction in IceCube and the measurement of the astrophysical neutrino flavor ratio sensitive to all flavors. It uses the high-energy starting events collected between 2010 and 2016 in the energy range between $60 \mathrm{TeV}$ and $10 \mathrm{PeV}$ deposited electromagneticequivalent energy. The sensitivity increases by identifying tau neutrino events with a decay length as low as $\sim 20 \mathrm{~m}$ and a deposited electromagnetic-equivalent energy above $\sim 100 \mathrm{TeV}$. In Section 2 the well-known data sample of high-energy starting events is revisited and the reconstruction of double cascade events is described. In Section 3 the observables, analysis method and systematic uncertainties are explained. The results are discussed in Section 4 and summarized in Section 5.

\section{Data Sample and Event Reconstruction}

The data sample used for this analysis is the high-energy starting event selection (HESE) [2]. Neutrino events are identified by defining the outer detector boundary as a veto region and by requiring that no more than 3 of the first 250 photoelectrons occur within the veto. In addition, at least 6000 photoelectrons are required per event corresponding to an energy threshold of $\sim 30 \mathrm{TeV}$ deposited electromagnetic-equivalent energy. Recently this event selection was updated to include a total of six years of data and now consists of 82 events [5]. Above a deposited energy of $60 \mathrm{TeV}$ considered for this analysis there are 49 events on a total estimated atmospheric background of $9.0_{-1.9}^{+3.4}$.

Three starting event topologies are considered (see Figure 1).

Single cascades are produced in electron-neutrino and all-flavor neutral current interactions. Double cascades are only produced in charged current tau neutrino interactions where the tau lepton decay produces either a hadronic or an electromagnetic cascade. Tracks are produced by muons in charged current muon neutrino interactions, in charged current tau neutrino interactions where the tau lepton decays into a muon and by muons from atmospheric air showers that do not trigger the veto condition.

Reconstruction of cascade- and track-like events is well established in IceCube [16]. An event is reconstructed using a maximumlikelihood fit where the expected arrival time distribution of photoelectrons from a hypothesis is compared to the observed distribution

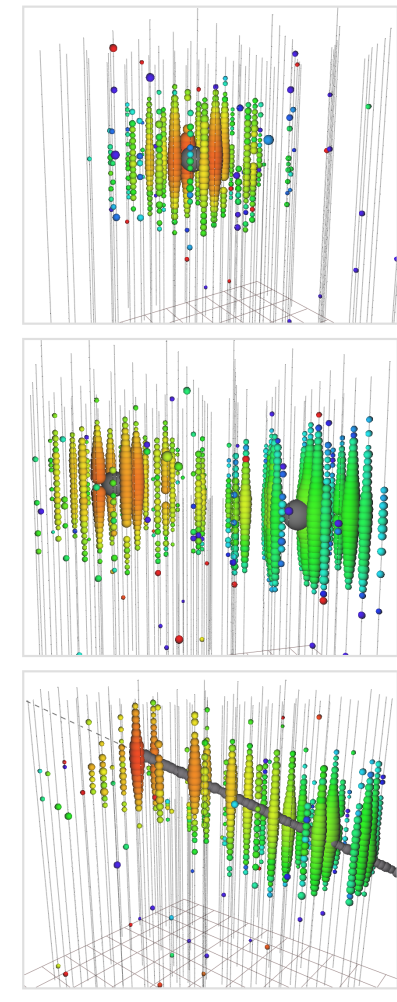

Figure 1: Simulated starting event topologies: single cascade (top), double cascade (middle) and track (bottom). at each DOM. For the first time double cascade events are explicitly reconstructed. The event hypothesis is constructed by extending a single cascade hypothesis by two more parameters: the decay length and the electromagnetic-equivalent energy deposited in the decay. Consequently, the second (decay) cascade is related to the first (interaction) cascade by assuming the two are connected by a particle traveling at the speed of light and in the same direction as the primary neutrino. Both assumptions are perfectly reasonable considering the energy scale and resolution of IceCube. 


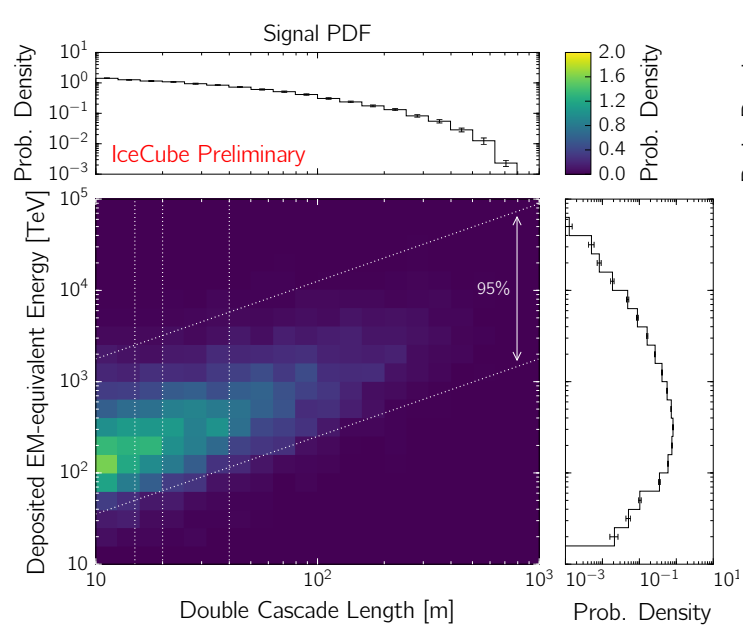

(a)

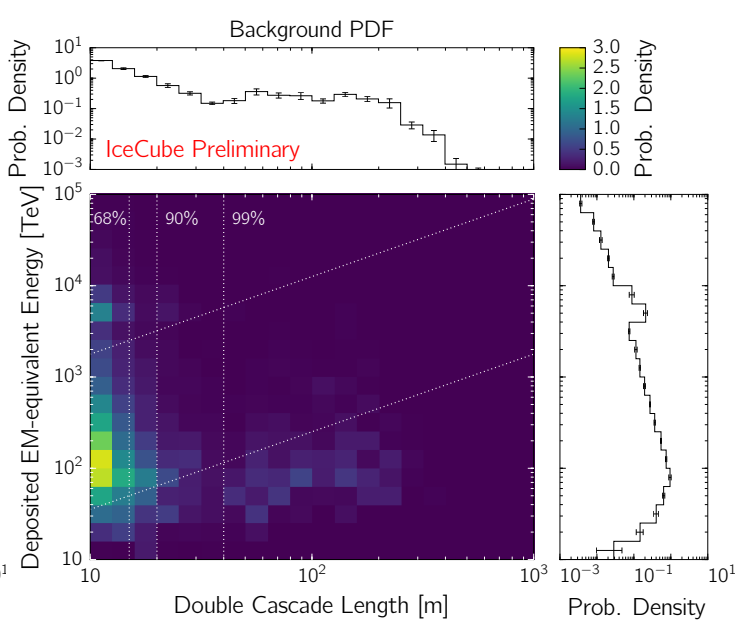

(b)

Figure 2: Probability density distributions in the double cascade sample for (a) charged current tau neutrino events where the tau lepton decay produces either a hadronic or an electromagnetic cascade and (b) for all remaining events including atmospheric and astrophysical backgrounds assuming an $E_{\nu}^{-2.3}$ astrophysical neutrino flux. The observables are the total deposited electromagnetic-equivalent energy and the double cascade length. They show a linear correlation for signal events due to the energy dependence of the mean decay length of the tau lepton. The diagonal lines indicate the $95 \%$ signal containment and the vertical lines indicate the $68 \%, 90 \%$ and $99 \%$ background exclusion, respectively. The dominant background close to the threshold of $10 \mathrm{~m}$ double cascade length are misreconstructed single cascades.

Each event is reconstructed assuming all three event hypotheses. If the best-fit of the double cascade reconstruction does not result in both cascades having at least $1 \mathrm{TeV}$ deposited energy and a vertex with a maximum distance of $50 \mathrm{~m}$ outside the fiducial volume, the event is classified as single cascade or track based on which hypothesis has a greater likelihood. For all remaining double cascade candidate events two topology estimators are defined on the basis of the reconstructed vertex positions and energies of the double cascade event hypothesis. The energy asymmetry $E_{\mathrm{A}}=\left(E_{1}-E_{2}\right) /\left(E_{1}+E_{2}\right)$ quantifies how much of the total deposited energy can be attributed either to the first or the second vertex. An energy asymmetry of \pm 1 means that the event is a single cascade by definition. The energy confinement $E_{\mathrm{C}}=\left(E_{\mathrm{C}, 1}+E_{\mathrm{C}, 2}\right) / E_{\text {tot }}$ gives the relation between the deposited energy $E_{\mathrm{C}, i}$ that is confined to a region of $\pm 40 \mathrm{~m}$ around the vertex position of each cascade $i=1,2$ and the total deposited energy $E_{\text {tot }}$ that is obtained from the deconvolution of all possible energy losses along the track hypothesis. An energy confinement close to 1 means that the event is very likely either a single or a double cascade. Events are classified as tracks by requiring $E_{\mathrm{C}}<0.99$. Double cascade events are selected by requiring a length greater than $10 \mathrm{~m}, E_{\mathrm{C}} \geq 0.99$ and $-0.98 \leq E_{\mathrm{A}} \leq 0.3$. All remaining events are classified as single cascades. Consequently, a ternary particle id (PID) is constructed using this classification scheme for three event topologies.

The total deposited energy and the decay length are used as observable for all events classified as double cascades by this PID selection method (see Figure 2). Although the deposited energy is not a direct measure of the tau energy the correlation between the mean decay length and the energy of the tau lepton (c.f. Section 1) also holds for the total deposited energy. The correlation of the total deposited energy and the decay length is clearly visible for the signal distribution. Since the 
decay length of the tau lepton scales with its energy and the astrophysical neutrino flux is falling steeply with energy, most double cascade events in the six-year data sample are expected to look very similar to single cascades - in contrast to the event signature illustrated in Figure 1 (middle). In fact, only $\sim 25 \%$ of all tau neutrino events expected in the HESE data sample have a decay length greater than $10 \mathrm{~m}$. The mean selection efficiency of these events is $\sim 50 \%$. The average misidentification fraction for single cascades and tracks is $\sim 10 \%$ and the misidentification fraction for double cascades ranges from $\sim 50 \%$ to $\sim 5 \%$ depending on the tau decay length. Successfully identified tau neutrino events have a median resolution of $\sim 3 \mathrm{~m}$ decay length and a neutrino energy range from $\sim 200 \mathrm{TeV}$ to $\sim 10 \mathrm{PeV}$.

\section{Analysis}

The analysis method to measure the astrophysical neutrino flavor ratio is a binned maximumlikelihood fit where a sum of Monte Carlo templates each containing the observables for an astrophysical or an atmospheric flux component is varied until it best describes the experimental data. The likelihood is constructed using the Poisson probability for each observation bin and a Gaussian penalty factor for each nuisance parameter using prior knowledge of the systematic errors [13].

The observables used in the likelihood fit are the total deposited electromagnetic-equivalent energy and the zenith angle for the single cascade sample and the track sample. For the double cascade sample the total deposited electromagnetic-equivalent energy and the double cascade length are used. The dependence on the zenith angle is omitted in the double cascade sample due to limited statistics. It is used in the single cascade and track samples because it constrains the atmospheric background. In the double cascade sample, however, most of the background above $60 \mathrm{TeV}$ is of astrophysical origin, hence the loss of information is inconsequential.

The astrophysical neutrino flux is modeled as a single power-law without a spectral cut-off. The flavor normalizations $\phi_{v_{\mathrm{e}}}, \phi_{v_{\mu}}$ and $\phi_{v_{\tau}}$ as well as the common spectral index $\gamma$ are free parameters of the fit. The atmospheric muon flux is based on the pure-proton extragalactic composition of the H3a composition model [17] using SYBILL 2.1 as hadronic interaction model [18]. It is renormalized to the number of expected muons estimated from events that are tagged in the veto region of the detector as described in [2]. The atmospheric muon flux is modeled with a statistical error of $\pm 30 \%$ that is determined from the number of these tagged events in experimental data. The HKKMS06 model [19] is used to describe the conventional atmospheric neutrinos from the decay of pions and kaons and the ERS model [20] is used to describe the prompt atmospheric neutrinos from the decay of charmed mesons. Both models are modified to match newer measurements of the primary cosmic ray spectrum in the "knee" region based on the H3a composition model $[17,21]$. Furthermore, both atmospheric neutrino fluxes are modified with the generalized self-veto probability as described in [22] to account for the self-veto effect of atmospheric neutrinos accompanying the vetoed muons from the same cosmic ray induced air shower in the atmosphere. An uncertainty of $\pm 30 \%$ on the conventional atmospheric neutrino flux includes uncertainties of the cosmic ray spectrum and composition ( $\pi / \mathrm{K}$ ratio) and hadronic interactions models. Since no prompt neutrino flux has been observed so far the prior is set to zero and the uncertainty is estimated to be $+65 \%$ in units of ERS which is derived from the $90 \%$ upper prompt limit from the six-year diffuse muon neutrino observation [9]. 
An energy scale is introduced as a systematic nuisance parameter that accounts for a potential bias of $\pm 15 \%$ on the deposited electromagnetic-equivalent energy. It arises from a $\pm 10 \%$ uncertainty on the relative DOM efficiency and $\mathrm{a}_{-7 \%}^{+10 \%}$ uncertainty on the scattering and absorption of the ice model. An additional systematic nuisance parameter is an ice anisotropy scale that accounts for a potential bias on the double cascade length based on the modulation of the nominal ice scattering of ${ }_{-8 \%}^{+4 \%}$ known as ice anisotropy [23]. Its uncertainty is estimated to be $\pm 30 \%$.

The main parameters of interest for constraining the flavor ratio are the astrophysical flux normalizations $\phi_{v_{\mathrm{e}}}, \phi_{v_{\mu}}$ and $\phi_{v_{\tau}}$. Sensitivity calculations are based on a $1: 1: 1$ flavor ratio assuming an astrophysical per-flavor flux of $\phi_{v}\left(E_{v}\right)=1.5 \cdot 10^{-18}\left(E_{v} / 100 \mathrm{TeV}\right)^{-2.3} \mathrm{GeV}^{-1} \mathrm{~cm}^{-2} \mathrm{sr}^{-1} \mathrm{~s}^{-1}$ corresponding to the best-fit in [3]. The expected number of events in the double cascade sample for a six-year dataset with this spectrum is $2.318_{-0.029}^{+0.038}$ tau neutrino events on a total astrophysical and atmospheric background of $0.939_{-0.092}^{+0.219}$ events. The majority of tau neutrino events are not identifiable as such and are thus classified as single cascades for neutral current interactions or charged current interactions where the tau decay length is too short or as tracks if the tau lepton decays into a muon. The number of identifiable tau neutrino events is strongly dependent on the assumed astrophysical neutrino flux and can be significantly reduced by a steeper spectrum, a spectral cut-off or a more complex spectral shape.

The sensitivity to constrain the tau neutrino flux is defined as median detection significance of the bestfit hypothesis compared to the nullhypothesis where the tau neutrino flux is zero using a likelihood ratio test. Injecting the aforementioned astrophysical flux at a flavor ratio of $1: 1: 1$ and modeling the teststatistic distribution as a $\chi^{2}$ distribution with one degree of freedom under the assumption of Wilks' theorem [24] the median detection significance for a six-year dataset is $2.5 \sigma$. The all-flavor ratio sensitivity is derived from a two-dimensional profile likelihood scan comparing each test point to the best-fit hypothesis using a likelihood ratio test. The confi-

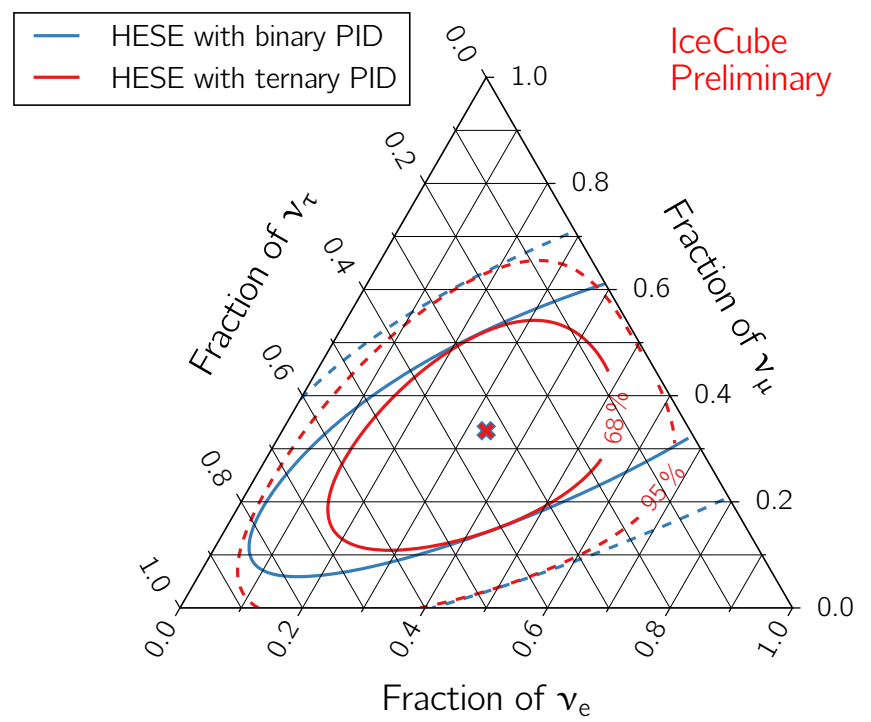

Figure 3: Median all-flavor ratio sensitivity using a binary PID of cascades and tracks (standard HESE) and a ternary PID of single cascades, double cascades and tracks (this work) dence levels are determined by modeling the test-statistic distribution as a $\chi^{2}$ distribution with two degrees of freedom. In Figure 3 this profile likelihood scan around the best-fit of the injected flavor ratio of $1: 1: 1$ is shown for a binary PID of cascades and tracks and a ternary PID of single cascades, double cascades and tracks using the Asimov dataset [25]. The binary PID corresponds to the method used in previous HESE publications and the ternary PID is the extension described here. It can clearly be seen how the degeneracy in the electron- and tau-neutrino fractions is decreased by the introduction of observables that are sensitive to the double cascade topology. 


\section{Results}

No tau neutrino candidate event with a deposited energy above $60 \mathrm{TeV}$ was observed. Consequently, the best-fit of the tau neutrino normalization is zero. The total astrophysical flux normalization and spectral index are consistent with the results discussed in [5]. The integrated $90 \%$ upper limit of the tau neutrino flux is $\phi_{v_{\tau}}\left(E_{\gamma_{\tau}}\right)<2.95 \cdot 10^{-18}\left(E_{\gamma_{\tau}} / 100 \mathrm{TeV}\right)^{-2.94} \mathrm{GeV}^{-1} \mathrm{~cm}^{-2} \mathrm{sr}^{-1} \mathrm{~s}^{-1}$. The best-fit astrophysical neutrino fractions are $f_{v_{\mathrm{e}}}=0.51_{-0.13}^{+0.12}, f_{v_{\mu}}=0.49_{-0.13}^{+0.12}$ and $f_{v_{\tau}}=0.00_{-0.00}^{+0.16}$ which correspond to a flavor ratio of $\sim 1: 1: 0$. A two-dimensional profile likelihood scan around the best-fit flavor ratio is shown in Figure 4. The flavor ratio of $\sim 1: 1: 1$ expected from pion decay at the source is in agreement with the best-fit. The previously published flavor ratio measurement is shown in comparison [13]. Note that these contours are smaller with respect to the electronand muon-neutrino fractions since they are derived from the combination of multiple data samples (including HESE) that contain a large number of track-like events. However, the tau neutrino fraction can be constrained better with the method presented here due to the increased sensitivity in the double cascade channel. This result will be included in a similar combined analysis in the future.

The lack of tau neutrino candidate events is best explained by either a statistical fluctuation or a spectral shape that is more complex than a single unbroken power-law. The updated HESE data sample including two more years revealed a lack of events with a deposited electromagnetic-equivalent energy above $200 \mathrm{TeV}$. The accumulation of more low-energy and fewer high-energy events might arise from a more complex spectral composition. However, it is not possible to distinguish more complex models from the assumed single power-law model with the currently available data. For a differ-

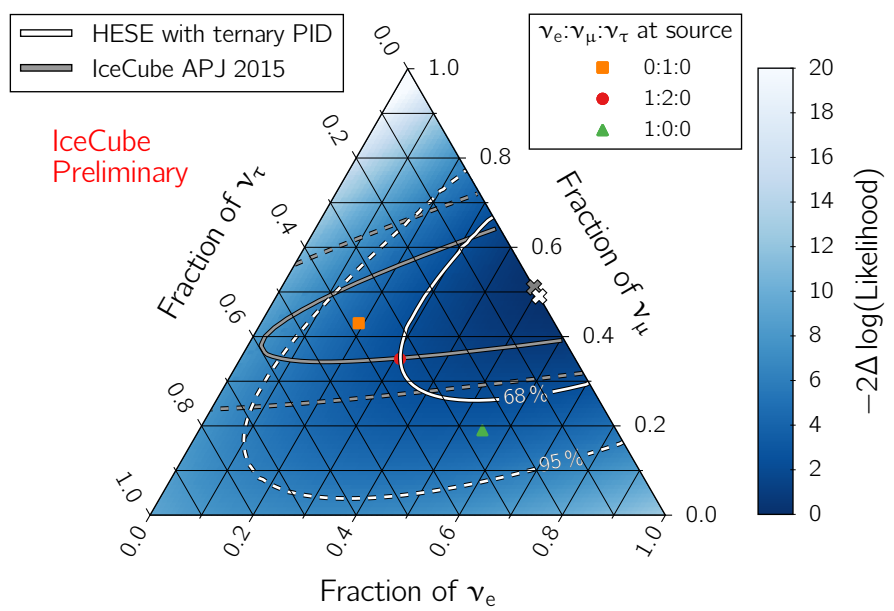

Figure 4: All-flavor ratio measurement using the ternary PID (this work) on the six-year HESE data sample. The best-fit is marked with ' $\times$ '. The solid and dashed lines show the $68 \%$ and 95\% contours, respectively. Compositions expected at Earth are marked for three different source scenarios. The gray lines show the previously published $68 \%$ and $95 \%$ contours [13].

ential flux model with reduced assumptions on the spectral shape by fitting an independent flux normalization in each energy band [5], the prediction of identifiable tau neutrino events is $1.441_{-0.018}^{+0.024}$ on a total background of $0.938_{-0.092}^{+0.219}$ events, assuming a flavor ratio of $1: 1: 1$. Not observing any tau neutrino candidate event then corresponds to a p-value of $9.3 \%$.

\section{Summary and Future Plans}

The work presented here extends the high-energy starting event analysis by introducing the reconstruction of a double cascade event hypothesis. On the basis of tau neutrino sensitive observ- 
ables a ternary PID is defined to measure the astrophysical flavor ratio and constrain the tau neutrino fraction with an improved sensitivity. No tau neutrino candidates were observed. The measured flavor ratio of $\sim 1: 1: 0$ is in agreement with an expectation of $\sim 1: 1: 1$ from pion decay at the source. There are multiple efforts to improve the constraints on the astrophysical neutrino flavor ratio by combining numerous event selections [26], by extending the double cascade reconstruction to events in the entire fiducial volume (including the veto region) [27] and by improving alternative detection methods of tau neutrino interactions [28].

\section{References}

[1] IceCube Collaboration, M. G. Aartsen et al., JINST 12 (2017) P03012.

[2] IceCube Collaboration, M. G. Aartsen et al., Science 342 (2013) 1242856.

[3] IceCube Collaboration, M. G. Aartsen et al., Phys. Rev. Lett. 113 (2014) 101101.

[4] IceCube Collaboration, PoS ( ICRC2015) 1081 (2016).

[5] IceCube Collaboration, POS ( ICRC2017) 0981 (these proceedings).

[6] H. Athar, C. S. Kim, and J. Lee, Modern Physics Letters A 21 (2006) 1049-1065.

[7] P. Lipari, M. Lusignoli, and D. Meloni, Phys. Rev. D 75 (Jun, 2007) 123005.

[8] M. Bustamante, J. F. Beacom, and W. Winter, Phys. Rev. Lett. 115 (2015) 161302.

[9] IceCube Collaboration, M. G. Aartsen et al., Astrophys. J. 833 (2016) 3.

[10] OPERA Collaboration, S. Dusini et al., AIP Conference Proceedings 1666 (2015) 110003.

[11] DONuT Collaboration, K. Kodama et al., Phys. Rev. D 78 (Sep, 2008) 052002.

[12] IceCube Collaboration, M. G. Aartsen et al., Phys. Rev. Lett. 114 (2015) 171102.

[13] IceCube Collaboration, M. G. Aartsen et al., Astrophys. J. 809 (2015) 98.

[14] IceCube Collaboration, M. G. Aartsen et al., Phys. Rev. D 93 (2016) 022001.

[15] IceCube Collaboration, PoS ( ICRC2015) 1066 (2016).

[16] IceCube Collaboration, M. G. Aartsen et al., JINST 9 (2014) P03009.

[17] T. K. Gaisser, Astropart. Phys. 35 (2012) 801-806.

[18] E.-J. Ahn, R. Engel, T. K. Gaisser, P. Lipari, and T. Stanev, Phys. Rev. D 80 (2009) 094003.

[19] M. Honda, T. Kajita, K. Kasahara, S. Midorikawa, and T. Sanuki, Phys. Rev. D 75 (2007) 043006.

[20] R. Enberg, M. H. Reno, and I. Sarcevic, Phys. Rev. D 78 (2008) 043005.

[21] IceCube Collaboration, M. G. Aartsen et al., Phys. Rev. D 89 (2014) 062007.

[22] T. K. Gaisser, K. Jero, A. Karle, and J. van Santen, Phys. Rev. D 90 (2014) 023009.

[23] IceCube Collaboration, Proceedings of ICRC2013 0580 (2014).

[24] S. S. Wilks, Annals Math. Statist. 9 (1938) 60-62.

[25] G. Cowan, K. Cranmer, E. Gross, and O. Vitells, Eur. Phys. J. C71 (2011) 1554.

[26] IceCube Collaboration, PoS (ICRC2017) 0976 (these proceedings).

[27] IceCube Collaboration, PoS (ICRC2017) 0973 (these proceedings).

[28] IceCube Collaboration, PoS (ICRC2017) 1009 (these proceedings). 